\title{
Marketing simbiótico. \\ Una aproximación al concepto desde una revisión teórica*
}

\author{
Recibido: 28 de noviembre de 2019 • Aprobado: 14 de diciembre de 2019 \\ https://doi.org/10.22395//seec.v22n53a5
Luz Alexandra Montoya-Restrepo**
Javier Sánchez- Torres ${ }^{* *}$
Iván Alonso Montoya-Restrepo ${ }^{* * * *}$

\section{RESUMEN}

Se presentan los principales aportes académicos del concepto marketing simbiótico, se expone la definición del concepto de diferentes autores desde sus primeros aportes. Esta presentación se realiza mediante una metodología de búsqueda conceptual en la que se establecen los principales autores y las tendencias de estudio del concepto. Como principal conclusión se encuentra que es un tema que, si bien ya se ha desarrollado, ha pasado por diferentes explicaciones que conllevan baja claridad en su definición, se limita así la unificación y constancia en el reconocimiento de los principales aportes de los autores, lo que evidencia la necesidad de consolidar las contribuciones y de esta manera, reconocer y fortalecer los estudios empíricos de esta.

\section{PALABRAS CLAVE}

Co-marketing, relaciones de cooperación, inter-colaboración, alianzas.

\section{CLASIFICACIÓN JEL}

M3, M30, M1

\section{CONTENIDO}

Introducción; 1. Metodología; 2. Marketing simbiótico; Conclusión; Referencias.

\footnotetext{
* Artículo derivado del Proyecto Hermes de la Universidad Nacional de Colombia, código: 44191, "Investigación en estrategias de Marketing: Gestión de Marca, love mark y neuromarketing", en el marco del grupo Management y marketing MEM, clasificación B GrupLAC Colciencias

* Administradora de empresas, magíster en Administración y doctora en Ciencias Económicas, Universidad Nacional de Colombia, Medellín, Colombia. Profesora titular de la Facultad de Minas, Universidad Nacional de Colombia, Medellín, Colombia. Correo electrónico: lamontoyar@unal.edu.co. Orcid: https://orcid. org/0000-0002-4896-1615

... Administrador de empresas, Universidad Nacional de Colombia, doctor en Business de la Universidad de Barcelona, Barcelona, España. Profesor y miembro del grupo de investigación Tetrix, Universidad de Medellín, Medellín, Colombia. Correo electrónico: jasanchez@udem.edu.co. Orcid: https://orcid. org/0000-0003-0959-3466

.... Administrador de empresas, magíster en Administración y doctor en Ciencias Económicas, Universidad Nacional de Colombia, Medellín, Colombia. Profesora titular de la Facultad de Ciencias Agrarias, Universidad Nacional de Colombia, Medellín, Colombia. Correo electrónico: iamontoyar@unal.edu.co. Orcid: https://orcid.org/0000-0002-8217-2177
} 


\title{
Symbiotic Marketing. Concept Approximation from a Theoretical Review
}

\begin{abstract}
The main academic contributions of the symbiotic marketing concept are presented, showing the definition different authors have given it from their first contributions. This is done through a conceptual search methodology in which main authors and trends of the concept study are established. The main conclusion is that although it has already been developed, symbiotic marketing has gone through different views that lead to low clarity in its definition and lack of unification and constancy in the recognition of the main contributions of authors, evidencing the need to consolidate the contributions and in this way, recognize and strengthen empirical studies.
\end{abstract}

\section{KEYWORDS}

Co-marketing, cooperative relationships, inter-cooperation, alliances.

\section{JEL CLASSIFICATION}

M3, M30, M1

\section{CONTENTS}

Introduction; 1. Methodology; 2. Symbiotic marketing; Conclusion; References.

\section{Marketing simbiótico. Uma aproximação para o conceito de uma revisão teórica}

\section{RESUMO}

São apresentadas as principais contribuições acadêmicas do conceito de marketing simbiótico, mostrando a definição do conceito de diferentes autores desde as primeiras contribuições. Isso é feito através de uma metodologia de busca conceitual na qual são estabelecidos os principais autores e as tendências do estudo conceitual. A principal conclusão é que é uma questão que, embora já tenha sido desenvolvida, passou por diferentes conceitos que levam a uma baixa clareza em sua própria definição, uma unificação e constância no reconhecimento das principais contribuições da os autores, evidenciando a necessidade de consolidar as contribuições e, dessa forma, reconhecer e fortalecer os estudos empíricos das mesmas.

\section{PALAVRAS CHAVE}

Co-marketing, relações de cooperação, inter-colaboração, alianças.

\section{CLASSIFICAÇÃO DE JEL}

M3, M30, M1

\section{CONTEÚDO}

Introdução; 1. Metodologia; 2. Marketing simbiótico; Conclusão; referências. 


\section{INTRODUCCIÓN}

Adler definió el marketing simbiótico por primera vez en 1966, el concepto se retomó en 1986 en el artículo "Symbiotic marketing revisited" de Varadarajan y Rajaratnam (1986), que destacó las oportunidades y características de los sistemas de cooperación entre las organizaciones para solucionar sus dificultades puntuales, en especial los de marketing. Sin embargo, el concepto de marketing simbiótico se ha trabajado muy poco en la literatura, se han utilizado más conceptos como alianza o de co-marketing, que enfatizan en el proceso de colaboración. Adler (1966) propuso como definición de marketing simbiótico "La alianza de recursos o programas entre dos o más organizaciones independientes, diseñado para mejorar el potencial de mercado de cada una de ellas" (p. 60). Señaló además una serie de oportunidades para aquellas organizaciones que lo implementaran, tales como:

- Acceso a nuevos y diferentes recursos de marketing

- Creación de nuevas vías de diversificación

- Sinergias en los gastos operativos

- Ingreso a nuevos mercados con riesgos relacionados y con recursos reducidos

- Actividades de marketing de mayor impacto dentro de presupuestos de marketing limitados

- Superación de las debilidades estratégicas mediante las fortalezas estratégicas de los demás

- Reducción de compromisos financieros en períodos de gestación del desarrollo de recursos operativos para la comercialización

- Estimulación del pensamiento ejecutivo al reunir a los grupos de gestión con diferentes habilidades, perspectivas y valores

- Mejora en las capacidades de negociación de las empresas participantes (Adler, 1966)

Venkataraman (1981) propuso el concepto marketing sinergy y lo definió como la preparación conjunta de programas y planes basados en recursos combinados de dos o más organizaciones independientes para lograr mayor productividad individual, más ventas y mayor satisfacción del cliente.

De igual manera, Varadarajan y Rajaratnam (1986) caracterizaron la naturaleza de las relaciones en el marketing simbiótico por medio de cinco dimensiones: 1) T= tiempo 
(largo plazo, medio plazo, corto plazo); 2) $\mathrm{P}=$ proximidad entre los participantes (longitud y cercanía); 3) N= nivel de cooperación (organizacional y funcional); 4) $\mathrm{E}=$ enfoque de relación (un solo simbionte o varios simbiontes); y 5) $\mathrm{A}=$ alcance a nivel de la función de marketing (formulación de una estrategia única o cooperación limitada a una función específica de marketing) que se pueden apreciar en el gráfico 1.

Gráfico 1. La dinámica del marketing simbiótico

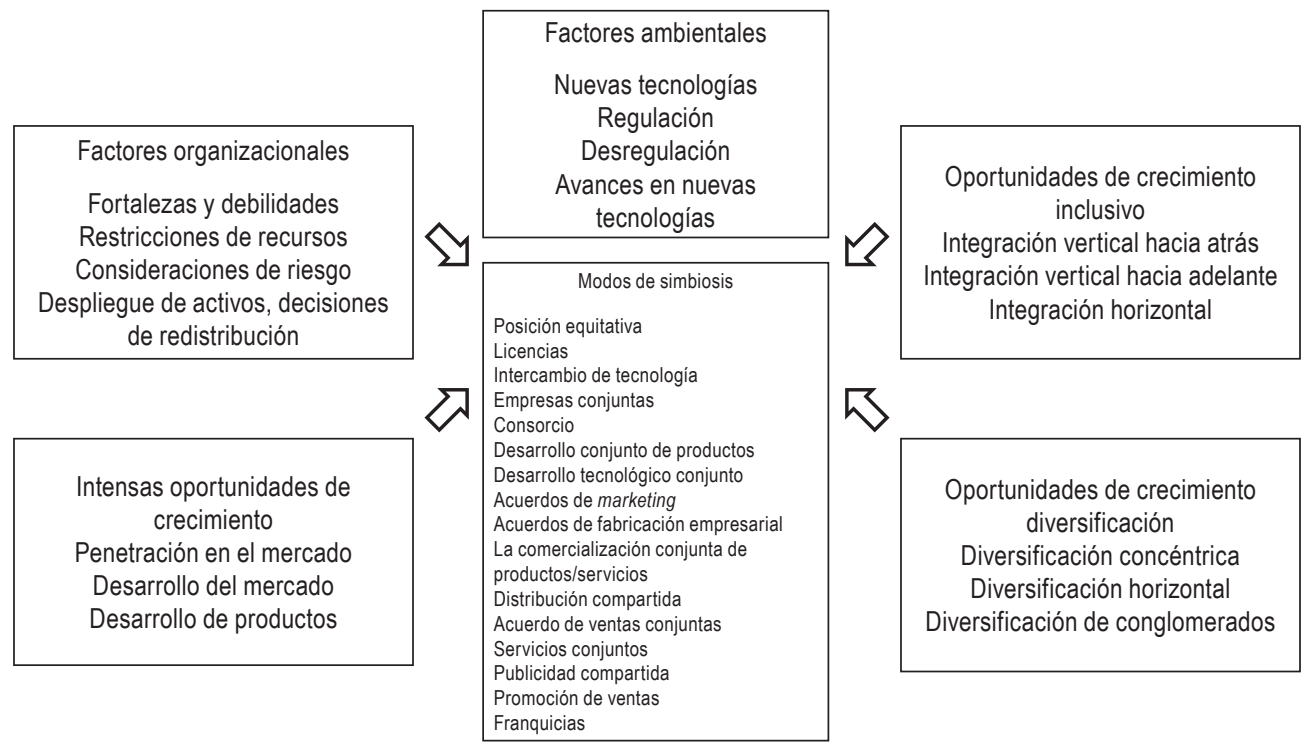

Fuente: adaptado de Varadarajan y Rajaratnam (1986)

Este gráfico señala cómo los factores organizaciones y ambientales, junto con las oportunidades de crecimiento, diversificación y crecimiento podrían llevar a diferentes modos de simbiosis que podrían generar modelos de acuerdos para alcanzar mayores ventajas competitivas, en especial, teniendo en cuenta la relación positiva para todas las organizaciones participantes.

Por su parte, Zaheer y Venkatraman (1995) desarrollan el concepto de mente colectiva para explicar cómo las interrelaciones de los miembros del equipo y la comprensión consciente funcionan dentro del sistema social. Bucklin y Sengupta (1993) proponen las alianzas en un entorno de co-marketing a partir de un modelo que permite la medición del desarrollo simbiótico en un entorno de eficiencia, proponen un modelo de dimensiones de un acuerdo bajo la metáfora de la simbiosis (gráfico 2). Este modelo de co-marketing propone una serie de relaciones positivas y negativas de acuerdo con la eficiencia o éxito de la integración, de tal forma que señala las 
variables que se deben tener en cuenta para que pueda ser oportuna la relación entre una o varias organizaciones.

\section{Gráfico 2. Modelo de co-marketing de Bucklin y Sengupta (1993)}

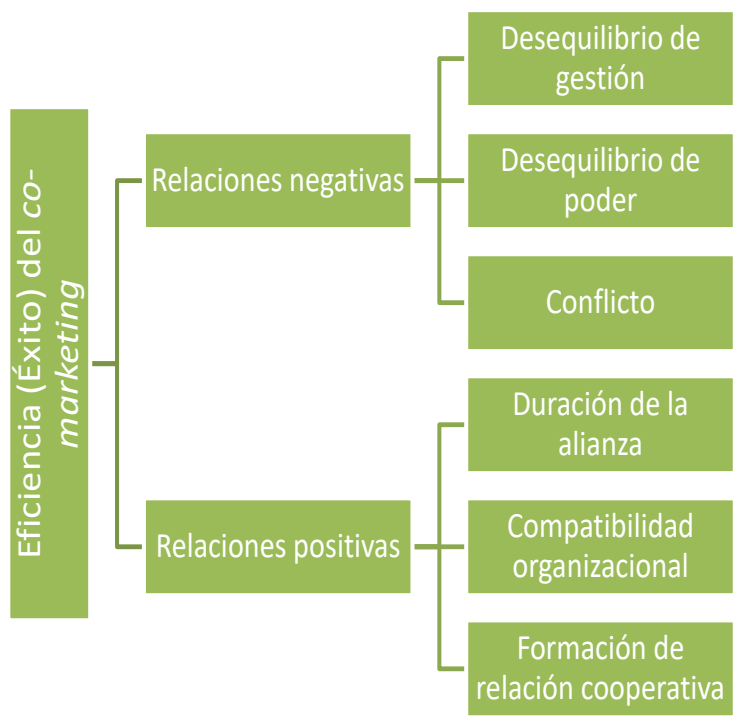

Fuente: adaptado de Bucklin y Sengupta (1993)

A pesar del avance conceptual realizado por diversos autores desde su concepción en 1966, y la utilidad del concepto desde la metáfora biológica aplicada al entendimiento organizacional, Montoya et al., (2010; 2012a; 2012b; 2016) encuentran que no se ha apropiado el concepto lo suficiente y por eso se realiza este esfuerzo por presentar los principales aportes de los autores en la temática.

El término simbiosis - derivado de la palabra griega compuesta "vivir juntos" o "vida en común"- se refiere a un fenómeno biológico en el que individuos de diferentes especies forman asociaciones persistentes de las que todos se benefician, los rasgos fundamentales de tal asociación se fundamentan en ser inter-específicos, cercanos y persistentes, lo que significa que deben ser organismos variados, de más de una especie, cuya interacción debe implicar contacto directo, y la relación que se busca tener es de larga duración. Además de los rasgos mencionados de inter-especificidad, cercanía y persistencia, una relación simbiótica estipula que el resultado de una interacción resulta en beneficios para cada uno de ellos. Este aspecto particular de la prestación, la aptitud o resultado es el atributo central para la identificación de los diferentes tipos de relaciones simbióticas o simbiosis con los tres tipos principales, denominados - de acuerdo con Moraitis (2019)- mutualismo, comensalismo y parasitismo. 


\section{METODOLOGÍA}

Para el análisis de los aportes conceptuales se utiliza la propuesta de Castellanos, Fuquene y Ramírez (2011) descrita en la figura 3. Esta metodología fue seleccionada por ser un método sistemático de análisis de tendencias a partir de la información disponible en los diferentes canales académicos. Es un método versátil, adaptable y flexible que permite hacer un barrido general del estado del arte a partir de las principales fuentes secundarias a través de cuatro fases. Esta metodología se centra en la definición de las necesidades de información a partir de un modelo de planeación, en la que se analiza una valoración de contenidos para la exploración. En la primera fase se organizan las necesidades de la investigación y se identifican los objetivos principales de la búsqueda. La segunda fase se encarga de buscar y descargar la información y se realiza una depuración de la información, se evalúan los documentos más importantes y con ellos, en una tercera fase se consolida la conceptualización con la revisión de las temáticas y conceptos de análisis. Esto para finalmente presentar los principales resultados encontrados en el desarrollo de este proceso.

\section{Gráfico 3. Metodología para el análisis de tendencias}

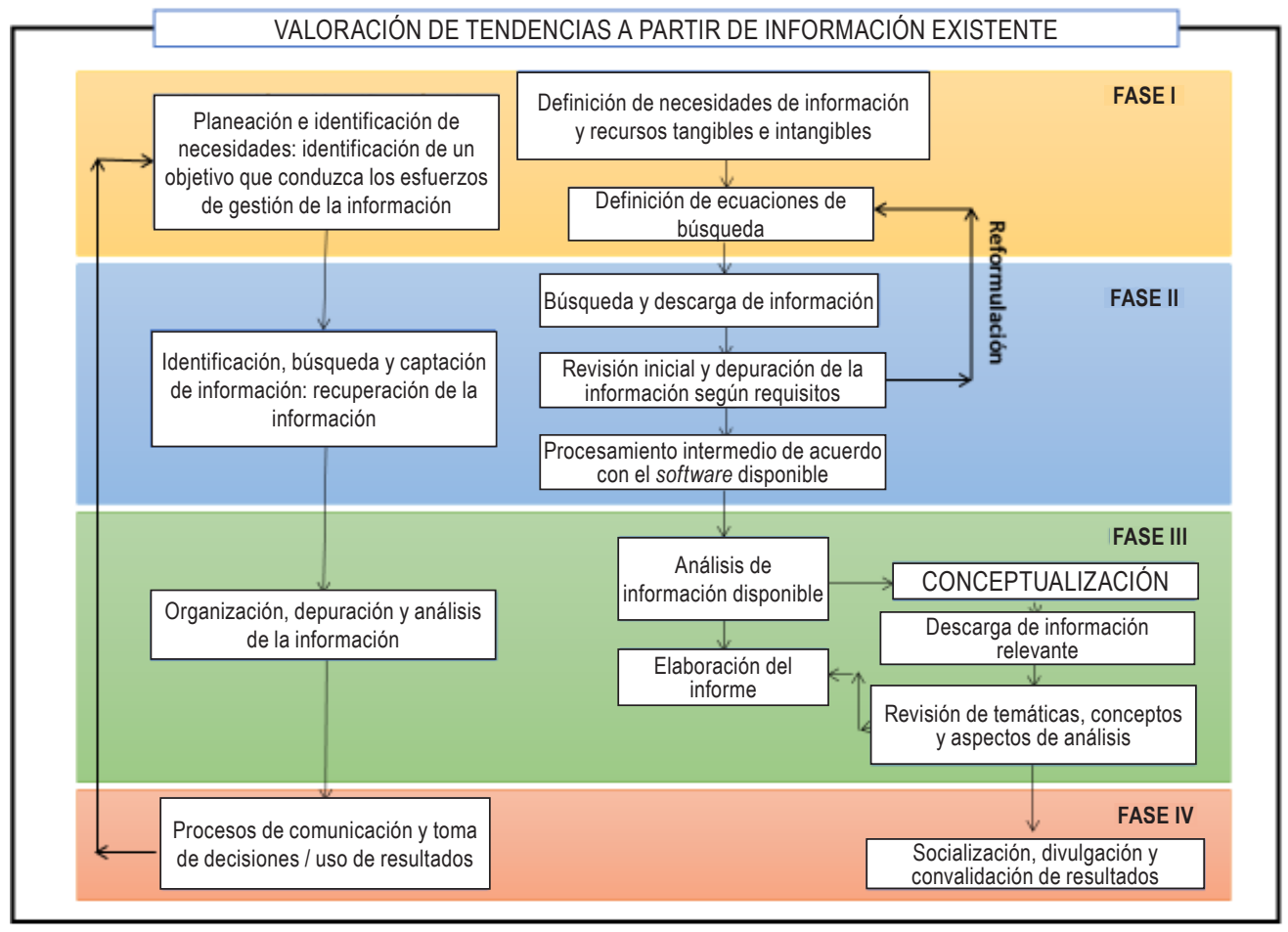

Fuente: Castellanos, Fuquene y Ramírez (2011) 
De acuerdo con esta propuesta metodológica, la primera actividad que se realizó fue buscar la información consolidada que presenta Scopus ${ }^{\circledR}$ con dos ecuaciones de búsqueda fundamentales. La primera se centró en los conceptos simbiosis y marketing, en el periodo 2011-2015 tuvieron un pico máximo de ocho documentos por año, los autores de más productividad presentan dos documentos relacionados.

El gráfico 4 proporciona información gráfica del desempeño que ha tenido el concepto desde 1969 en sus inicios; en general, se encuentra un crecimiento sostenido que ha tenido algunas caídas en la producción de documentos, esto se puede deber a que en las publicaciones hay tiempos "valle" mientras los manuscritos se escriben y someten a los procesos de publicación. Sin embargo, y pese a estos picos y caídas, hay documentos que mantienen la dinámica, lo cual indica que el concepto está vigente. Los autores más productivos solo han publicado dos artículos en el periodo.

Gráfico 4 cantidad de documentos y principales autores por año en Scopus de simbiosis y marketing
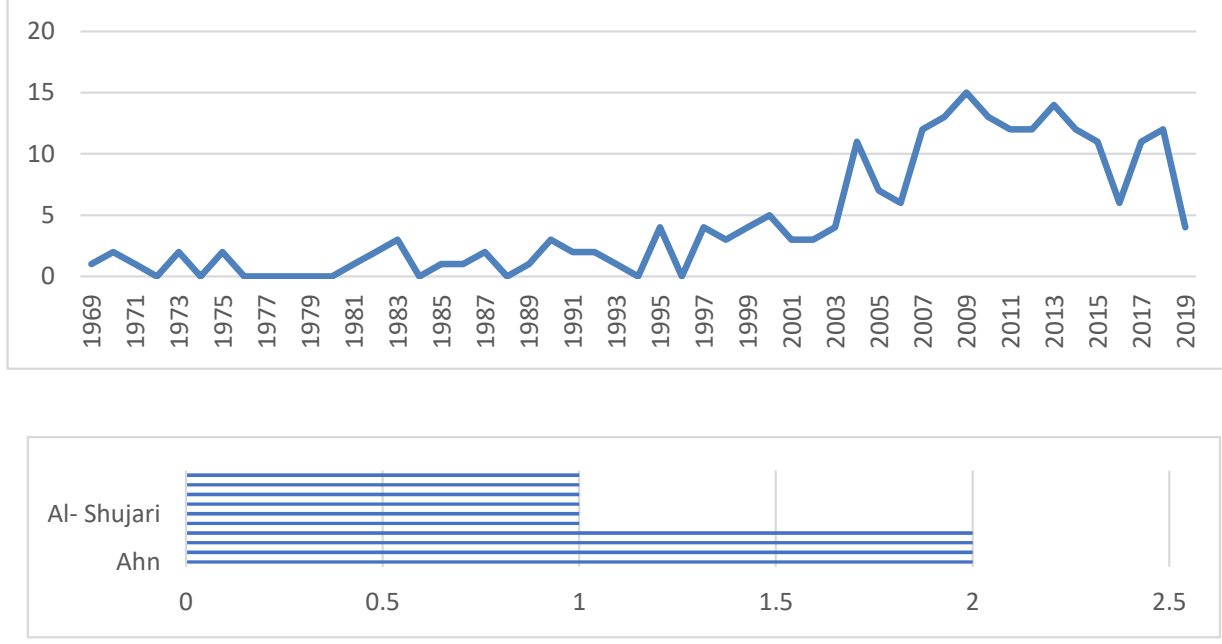

Fuente: elaborado a partir de resultados de búsqueda en Scopus

En cuanto al gráfico 5 , se puede señalar que los documentos se centraron en las áreas de negocios (19,4\%), ingeniería (12,4\%) y ciencias sociales (11,6\%).

Cuando se analiza la ecuación de co-marketing para la búsqueda se encuentran muchos más aportes de los autores -en especial de Estados Unidos-, con un crecimiento importante desde el 2010, que se puede observar en el gráfico 6, se pasa de un promedio de 5 documentos a un total de 37 documentos proyectados en el área en 2020, lo cual indica un crecimiento sostenido de desarrollo de esta área de comparación metafórica. 


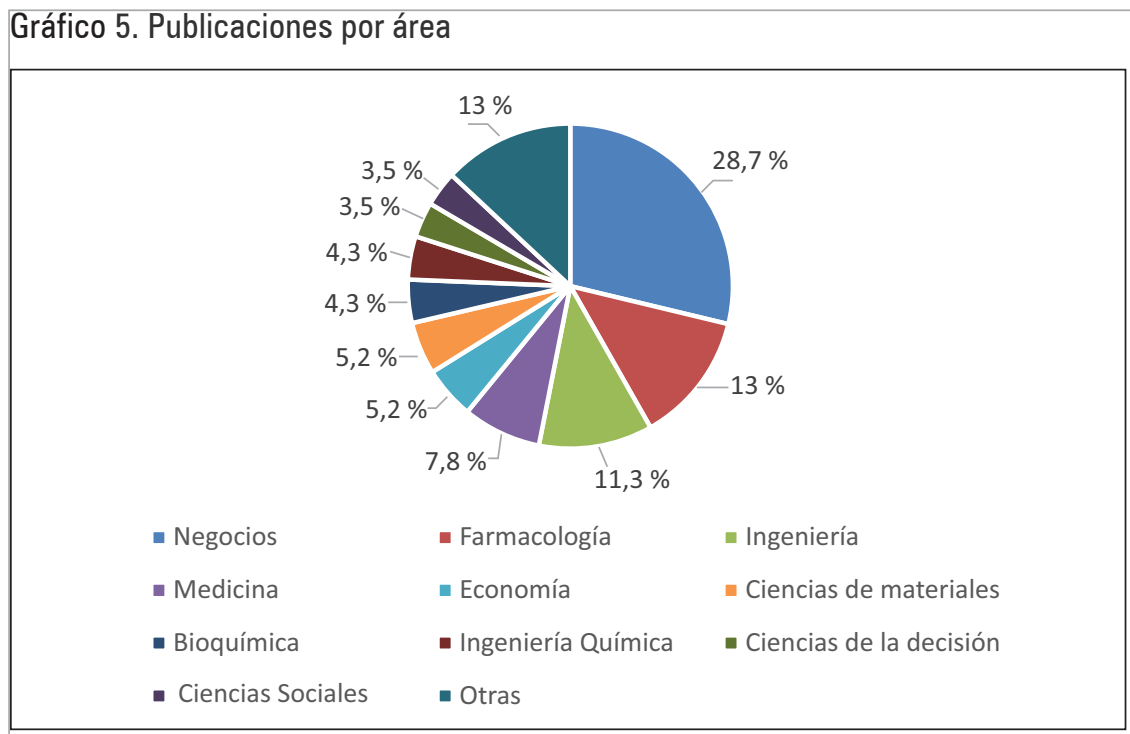

Fuente: elaborado a partir de resultados de búsqueda en Scopus

\section{Gráfico 6. Publicaciones sobre co-marketing por año}

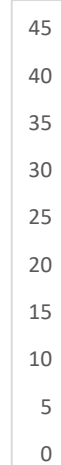

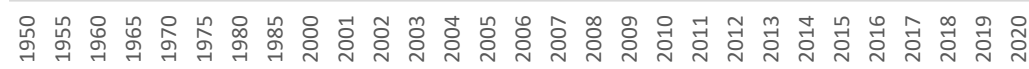

Fuente: elaborado a partir de los resultados de la búsqueda en Scopus

Finalmente, se presenta la ecuación alianza y marketing, en la que se encuentra presente el concepto, pero este no ha sido definido como simbiosis. En este sentido, la alianza proporciona los elementos necesarios para llegar a la simbiosis, el gráfico 7 demuestra el interés del desarrollo de estos conceptos, en especial en Estados Unidos en donde más de 600 documentos explican las oportunidades y dificultades de las relaciones, específicamente en el área de mercadeo. Se observa en esta interacción de alianza y marketing, un pico en la producción de documentos 
académicos muy importante en el 2004 con 170 documentos, seguido con 160 en el 2005 y 127 en 2003 y 2006. Desde el 2012 se evidencia un promedio de 52 artículos por año, lo cual denota que la contribución es significativa, pero no crece con motivación, a pesar de la importancia que ha retomado el tema de las alianzas estratégicas en las organizaciones.

Gráfico 7. Alliance + marketing

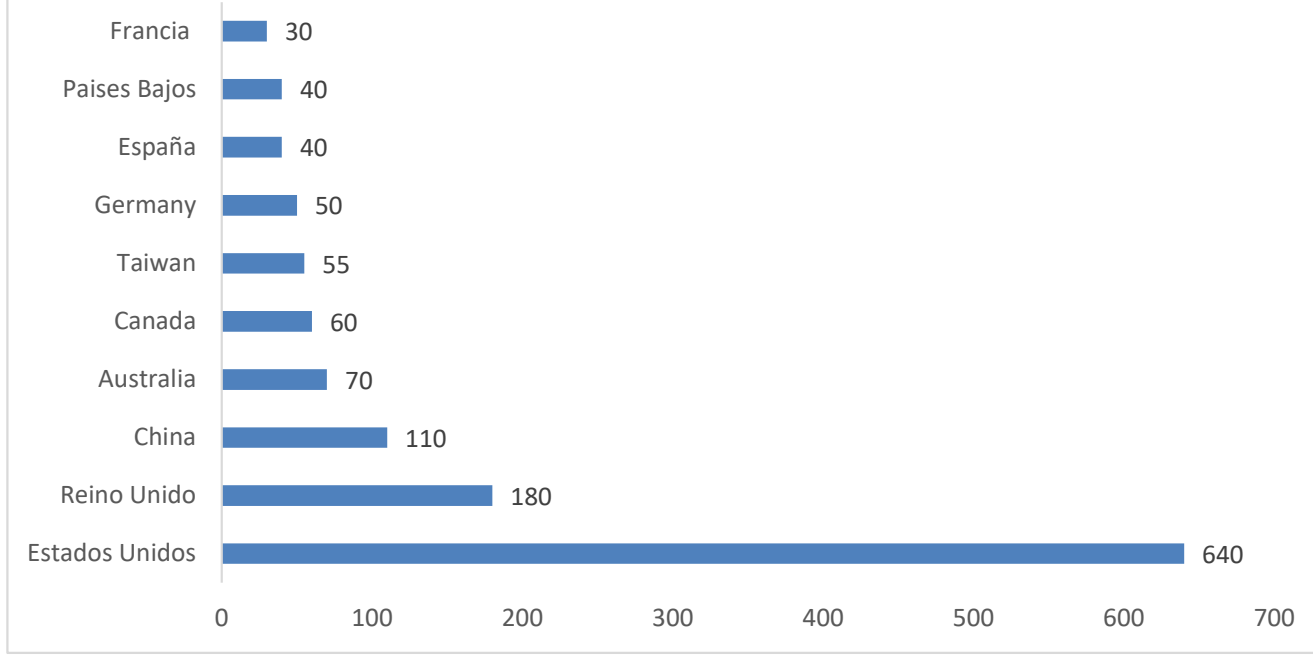

Fuente: elaborado a partir de los resultados de la búsqueda en Scopus

Con base en esta producción académica se propuso la tabla 1 que presenta a los principales autores del marketing simbiótico, incluye temas de co-marketing listados por año de publicación, inicia con el autor principal de la contribución.

Tabla 1. Autores principales del marketing simbiótico

\begin{tabular}{ll}
\hline \multicolumn{1}{c}{ Autor } & \multicolumn{1}{c}{ Descripción } \\
\hline Adler, (1966) & $\begin{array}{l}\text { El marketing simbiótico supone la alianza entre dos o más organizaciones in- } \\
\text { dependientes que comparten recursos para mejorar el potencial de mar- } \\
\text { keting de todas ellas. }\end{array}$ \\
\hline & $\begin{array}{l}\text { Son relaciones contractuales emprendidas por firmas cuyos productos res- } \\
\text { pectivos son complementos en el mercado. Están destinados a amplificar } \\
\text { Bucklin y Sengupta } \\
\text { (1993) }\end{array}$ \\
& $\begin{array}{l}\text { yentariedades, que implican la coordinación entre socios en uno o más } \\
\text { aspectos del marketing y se puede extender a la investigación, desarrollo de } \\
\text { productos e incluso producción. }\end{array}$ \\
\hline
\end{tabular}




\begin{tabular}{|c|c|}
\hline Autor & Descripción \\
\hline \multirow{9}{*}{ Udaya (1994) } & $\begin{array}{l}\text { En primer lugar, la formación de una relación simbiótica está diseñada para } \\
\text { devolver beneficios sinérgicos a las partes participantes. En segundo lugar, } \\
\text { la relación está formada por organizaciones independientes. Por lo tanto, } \\
\text { los acuerdos de entidades comerciales, como la integración vertical com- } \\
\text { pleta y el resultado de una fusión o adquisición, no son de naturaleza sim- } \\
\text { biótica. Se enfoca en las "organizaciones que continúan manteniendo su } \\
\text { identidad distinta y no están vinculadas por la tradicional en la relación de } \\
\text { intermediación entre mercadotecnia y marketing" (Varadarajan y Rajaratnam, } \\
\text { 1986, p. 8). Finalmente, las relaciones simbióticas se forman al combinar } \\
\text { recursos. El marketing simbiótico se define entonces como: }\end{array}$ \\
\hline & $\begin{array}{l}\text { [...] la combinación estratégica de recursos por parte de dos o más acto- } \\
\text { res, que pueden ser competidores directos u operar en mercados com- } \\
\text { pletamente distintos, para obtener rendimientos superiores del mercado } \\
\text { que ninguna de las partes puede alcanzar de forma independiente. (p. 10) }\end{array}$ \\
\hline & Características de un acuerdo simbiótico: \\
\hline & $\begin{array}{l}\text { 1. Las empresas son absolutamente independientes (ambos económica y } \\
\text { operacionalmente). }\end{array}$ \\
\hline & $\begin{array}{l}\text { 2. Las empresas participan en un acuerdo de marketing simbiótico de mane- } \\
\text { ra voluntaria. }\end{array}$ \\
\hline & $\begin{array}{l}\text { 3. Todas las empresas participantes mantienen su organización e identi- } \\
\text { dades del mercado, durante e incluso después del período del acuer- } \\
\text { do. Las relaciones simbióticas son importantes para las organizaciones } \\
\text { porque desarrollan ventajas competitivas que pueden no haber sido po- } \\
\text { sibles sin la contribución de los recursos de cada empresa. Beneficios } \\
\text { del marketing simbiótico: }\end{array}$ \\
\hline & 1. Acceso a recursos de marketing nuevos y establecidos \\
\hline & 2. Superación de las debilidades estratégicas \\
\hline & 3. Reducción de compromisos financieros \\
\hline
\end{tabular}

Las relaciones simbióticas son importantes para las organizaciones por varias razones, la más importante de ellas es que mediante el desarrollo de la cooperación se desarrollan ventajas que pueden no haber sido posibles sin la contribución de cada recurso de la firma. Hay cinco resultados del marketing simbiótico (denominado red de Doyle) que contribuyen a esta ventaja competitiva mejorada (Doyle, 1995):

Doyle (1995) 1 . Al centrarse en toda la cadena de valor, se tiene la oportunidad de mejorar la calidad o reducir los costos antes de la simbiosis; 2. Cada empresa tiene acceso a conocimientos técnicos externos que son cruciales para la innovación de productos y procesos de reingeniería; 3. Las alianzas simbióticas también permiten que las empresas sean más dinámicas. Las empresas pueden acceder a recursos que puede que no se hayan desarrollado internamente de forma inmediata al ubicar socios con dichos recursos; 
Autor

Descripción

4. Se ofrece un efecto en el apalancamiento financiero de una empresa que depende de la naturaleza de la alianza, una empresa puede aumentar los

Doyle (1995) ingresos, el rendimiento de los activos y convertir los costos fijos en variables para producir así un ingreso neto anual más estable; 5. Cada empresa puede reducir sus costos y recibir un producto de mayor calidad.

Se manifiestan como estrategias cooperativas interorganizacionales que Varadarajan y Cunningham (1995) implican la puesta en común de recursos y habilidades específicas para alcanzar objetivos comunes: obtener acceso a nuevos mercados; fortalecer nuevos negocios y competir con costos. Proporcionan un marco de información para las alianzas.

El proceso a través del cual dos o más socios desarrollan algunas iniciati-

Cherubini (1999) vas analíticas, estratégicas u operativas para alcanzar los objetivos de marketing a través de la satisfacción y el conocimiento del cliente.

Identifica las alianzas de marca como una de las corrientes de investigación

Venkatesh et al., (2000) de estudios de co-marketing en el transporte aéreo. Especifíca la importancia de obtener beneficios para el fortalecimiento de las marcas.

Diferencia el co-marketing del co-branding, subraya las diferencias entre estos conceptos al considerar el segundo como una especificación de la pri-

De Vita (2000) mera. Señala el co-marketing como el desarrollo de un objetivo de mercado, mientras que el co-branding se centra en la visibilidad de la marca y su fortalecimiento.

Lockett y Blackman (2001)

La estrategia simbiótica como organización permite los contactos intrapersonales y fortalece la conexión entre diferentes enlaces como elemento fundamental de la relación.

Cegarra y Michel (2003)

Proponen tres tipologías específicas de alianzas en el concepto de co-marketing: 1) co-desarrollo de productos o procesos productivos, 2) promoción conjunta de productos existentes, 3) promoción conjunta de la co-marca.

En el marketing simbiótico la estrategia de empresas colaborativas para el trabajo en red permite alcanzar objetivos estratégicos y se define como la

Shimizu (2003) unión formal de atracción de firmas independientes para el aprovechamiento de sus recursos combinados, destinados a ser utilizados contra otras firmas específicas y mejorar la competitividad.

Se le caracteriza por la presencia de dos o más socios, su objetivo es generar ventajas y beneficios sinérgicos para todos los agentes involucrados.

Iasevoli (2004) Propone procesos de gestión en los cuales se organizan, planifican y evalúan las actividades; también se implementan alianzas en todas las actividades de marketing (analíticas, estratégicas, operativas) para alcanzar un aumento en el valor percibido para los clientes.

Las firmas eligen qué segmentos de consumo y sus canales de distribución, se busca que las empresas y los consumidores converjan. Se propone un Prahalad y Ramaswamy (2004) valor co-creado en múltiples puntos de interacción. La base del valor es la experiencia de co-creación. Los consumidores y las comunidades de consumidores también pueden iniciar un diálogo entre ellos. 
Autor

Descripción

El consumidor es quien elige la firma nodal y el entorno de experiencia

Prahalad y Ramaswamy (2004) para interactuar con y co-crear valor. La firma nodal, sus productos y servicios, empleados, múltiples canales y comunidades de consumidores vienen conjuntamente para constituir el entorno de experiencia para individuos para co-construir sus propias experiencias.

Las alianzas de co-marketing se consideran un tipo de alianza estratégi-

Thoumrungroje y

Tansuhaj (2004) ca. Se realizan para promover la competitividad como estrategia horizontal que permite aumentar los recursos y obtener así un mejor valor agregado en los productos.

Farrelly y Quester (2005) Las alianzas de co-marketing son relaciones que buscan comercializar productos o servicios compartiendo el producto, la marca o la imagen corporativa.

Ahn, Kim y Forney (2010) Una alianza de co-marketing se refiere a la colaboración lateral entre empresas de diferentes sectores. Los participantes están en el mismo nivel en la cadena de valor añadido, por lo que les permite crear denominación conjunta y cooperar técnicamente.

En los casos en los que las industrias experimentan disminuciones en la Taek Yi, Lee y Dubinsky (2010) rentabilidad, las alianzas de co-marketing se han convertido en un medio popular para que las empresas mejoren sus ventas y rentabilidad en las industrias maduras, esta investigación proporciona elementos cognitivos acerca de las alianzas.

El "acuerdo simbiótico" es una relación en la cual se proponen nuevos mo-

Hansen (2011) dos de producción, estos destacan que el interés común debe ser más importante que el particular y buscan que las organizaciones que participan tengan respuesta a sus propios intereses a partir de la relación establecida.

Señala que el marketing simbiótico no ha prestado suficiente atención a los efectos de orientación de mercado y capital social, proponen cómo se Huang et al. (2011) debería considerar para investigaciones futuras, así como la necesidad de desarrollar mejores metodologías de análisis para entender los procesos de confianza y alianzas estratégicas.

Las empresas pueden reducir su exposición al riesgo de mercado al distribuir dicho riesgo entre todos los miembros individuales de la relación simbiótica. Esto es especialmente crítico en los campos de tecnología emergente que desarrollan productos como microprocesadores de computadora de alta velocidad, investigación genética y televisión de alta definición. Finalmente, el establecimiento de relaciones simbióticas se puede entender como estrategia defensiva para combatir potenciales adquisiciones y competidores corporativos hostiles. Al formar relaciones simbióticas fuertes y diversificadas, las empresas elevan efectivamente las barreras de entrada a cada mercado que penetran (Dickinson, 2012, p. 14).

Otros beneficios son:

- Intercambio de instalaciones, licencias y sociedades conjuntas de franquicia

- Posición de equidad de licencia 


\begin{tabular}{ll}
\hline Autor & \\
\hline - Intercambio de tecnología de franquicias \\
- Cooperativas o empresas conjuntas del consorcio \\
- Desarrollo de producto conjunto de integración vertical administrada \\
- Canales de comercialización tradicionales \\
Dickinson (2012) \\
- Desarrollo tecnológico conjunto \\
- Acuerdos de importación y exportación \\
- Acuerdos de comercialización conjunta \\
- Acuerdos de fabricación \\
- Organizribución compartida \\
- Departamento de servicio conjunto
\end{tabular}

Se introduce un modelo matemático de simbiosis entre diferentes especies teniendo en cuenta la influencia de cada especie en las capacidades de transporte de las otras. Las entidades modeladas pueden pertenecer a sociedades biológicas y ecológicas o a sociedades sociales, económicas

Vyacheslav Yukalov y Sornette (2012)

Kiran y Mousumi (2013) recursos, de los cuales al menos uno es de marketing, se intercambian
mutuamente entre los miembros y los beneficios que se acumulan se
dan en diferentes áreas funcionales, de las cuales al menos una conlleva
un impacto positivo directo en su potencial de marketing. Cuando hablamos de simbiosis, estamos buscando la posibilidad de la propuesta de valor final para un cliente, que es impracticable de producir solo. Consideramos el modelo de simbiosis como un concepto evolutivo

\section{Shaw}

(2013) importante y un enfoque estratégico para crear valor colaborativo que sería imposible de ofrecer de manera autónoma. La idea de la simbiosis entre el cliente y la empresa se puede describir con niveles de sinergia: centrado en el producto y simbiosis centrada en el cliente.

Exploran la colaboración a través del concepto de simbiosis, el cual reconoce que diferentes organismos pueden formar asociaciones persistentes Thomas y Wind (2013) o colaboraciones que benefician a los involucrados en diversos grados. Proponen un modelo de simbiosis para ayudar a entender cómo el equilibrio dinámico entre los participantes puede contribuir a diferentes tipos de innovación. Proponen la colaboración interna y externa. En la interna se 
Autor

Thomas y Wind (2013)

Grieco, y Lasevoli

(2017)

Donato, Farina y

Donaire (2017)

confianza entre ellos.

Las relaciones simbióticas no se limitan a la biología y la ecología: el término "simbiosis" también se utiliza para describir las relaciones económicas o financieras, ha sido empleado en trabajos de investigación que estudian colaboraciones de innovación y participación de los usuarios en los procesos de innovación, las cuales se consideran una relación: los clientes ayudan a las empresas a identificar necesidades latentes y nuevos valores, maximizan así sus posibilidades de tener acceso a los productos terminados que realmente necesitan.

Proponen la brújula del modelo de negocio compartido, la cual cuenta con seis dimensiones clave para la colaboración de los modelos de negocio, Muñoz y Cohen (2018) cada una va acompañadas de tres opciones diferentes. La brújula podría generar más de 100 permutaciones diferentes de combinaciones de modelos de negocio.

La estrategia simbiótica ayuda a los socios comerciales simbiontes a superar sus limitaciones de recursos de manera más eficiente para reducir la amenaza del mercado, competencia e incertidumbre, y para lograr una cooperación a largo plazo y un crecimiento sostenible. Como resultado,

Li et al. (2018) todos los socios o simbiontes pueden rendir mejor que los que participan en otros tipos de alianzas estratégicas. Aquí, la razón principal es que, en otros tipos de alianza estratégica distintas a la simbiótica, los socios involucrados todavía pueden tener intereses contradictorios o luchas de poder entre ellos con efectos negativos en sus actuaciones (Li et al., 2018, p. 122).

A través de la ciencia evolutiva de la "teoría de la endosimbiosis" interpretan las interdependencias del empleo juvenil y el turismo. Proponen tres clasificaciones de simbiosis: mutualismo, comensalismo y parasitismo para:

Robinson et al., (2019) poner a prueba la utilidad analítica de este enfoque teórico como medio para desempacar las relaciones laborales entre jóvenes trabajadores y turismo, unificar la literatura -hasta ahora dispersa- sobre la relación laboral entre la juventud y el turismo. En particular, modela un valor explicativo 
Autor

Robinson et al., (2019)

Cap et al., (2019)

Skarzauskiene

Maciuliene e Ivanova-

Radovanova (2019)

Ketonen-Oksi y

Valkokari (2019)

Acquire, Carbone y

Massé (2019)

y Hewedi (2019)

\section{Descripción}

de la teoría de la endosimbiosis al navegar por las éticas y moralidades de las ciencias sociales en el progreso de la comprensión de la intersección entre los trabajadores del turismo y los jóvenes.

La colaboración en redes interorganizacionales es un motor importante de innovación. Sin embargo, los métodos con los cuales los profesionales pueden confiar para gestionar las redes de innovación siguen siendo escasos. Esta investigación llena dicho vacío al proporcionar un enfoque que aumenta la producción de innovación al intensificar la colaboración en las redes. Se adoptó una metodología de investigación de acción con dos ciclos de acción. El primer ciclo desarrolla un método de evaluación de la red diseñado para evaluar el potencial de colaboración de una red. El segundo ciclo se basa iterativamente en el primer ciclo y amplía el método mediante un enfoque de gestión de la red a nivel individual.

Definen la co-creación como la participación de los ciudadanos en el proceso de iniciación y/o diseño de los servicios públicos con el fin de (co) crear resultados y valor beneficiosos para la sociedad. Los espacios públicos abiertos mediados son entornos ideales para la co-creación, debido a la participación de la comunidad y las TIC en la creación de conocimiento. Las ideas del ejercicio empírico permiten extraer recomendaciones gerenciales y organizativas para fortalecer a los ciudadanos, desarrolladores de TIC, instituciones públicas y gubernamentales en la creación de espacios públicos abiertos, inclusivos y reflexivos.

Las invenciones más importantes surgen de escuchar a las personas y están impulsadas por la colaboración, lo que permite invenciones colectivas que requieren tolerancia y diversidad.

Los mecanismos de cooperación se apalancan en socios internos y externos, lo cual permite crear nuevos recursos, desarrollar una comunidad y diversificar los negocios de las organizaciones a partir de nuevos productos y servicios. Los beneficios potenciales se centran en la diversificación de los mercados, mejoramiento de la reputación de las organizaciones, incremento en el servicio a los clientes, desarrollo de una cultura en los empleados de motivación y promoción de la innovación; supone aprovechar nuevos modelos de negocios, construir comunidades de marca asociados a valores sociales y ambientales.

A través de la investigación entienden la forma dinámica y moderna de cocrear innovación valiosa en el campo del marketing, en la promoción actualizada de destinos, y otros sectores como las empresas aéreas. El documento destaca la capacidad moderna de conectar y promover a las partes interesadas dentro de un marco cada vez mayor del entorno globalizado.

El marketing cooperativo es un término utilizado para describir la relación entre las empresas para promover sus ventas y servicios, en este destacan conceptos como colaboración, coordinación, alianzas, networking, sociedades y las co-marca. El objetivo del marketing cooperativo es desarrollar un entorno de tareas colectivas que reúna a las organizaciones para 
Autor

Abou-Shouk, Tamam, y Hewedi (2019)

Moraitis (2019

(a)

Pinarbasi (2020)

Lorek y Lorek (2020)

Mikulášková et al. (2020)

\section{Descripción}

mejorar su capacidad para desarrollar conjuntamente el mercado y competir en el mercado capitalizando oportunidades y el fomento del conocimiento de la marca.

Propone la simbiosis como un modo multidisciplinario mediante el cual las disciplinas que interactúan son complementarias y ejercen influencia entre sí, pero siguen siendo distintas e identificables.

La economía compartida ha surgido como un modelo económico influyente en las últimas décadas. Una literatura considerable ha crecido en torno a los subtemas de la economía colaborativa, incluidos los estudios relacionados con las empresas, los relacionados con el sistema y otros estudios relacionados con el contexto. Propone tres niveles para el estudio: nivel de consumo, nivel de negocio y de marca, y niveles de sistema.

Proponen el concepto de economía circular, este requiere de transformaciones en el campo de la competencia, desarrollo, innovaciones y gobernanza organizacional, así como la conciencia pública. La economía circular crea oportunidades para lograr beneficios en la economía (la eficiencia de los procesos de producción, innovaciones, seguridad energética), así como en los ámbitos social y ambiental (da forma a la seguridad ecológica).

Proponen el modelo de gestión en espiral y sus principios se basan en la existencia exitosa a largo plazo de sistemas vivos. Los principios se pueden aplicar a la gestión empresarial y organizativa. Los sistemas vivos gestionan el éxito a largo plazo mediante la adquisición de energía de su entorno (a través de la nutrición), mientras que las empresas adquieren la energía de los clientes y empleados con el fin de eliminar la entropía, que en las empresas se manifiestan como pérdidas económicas. La gestión en espiral se basa en la sinergia de los sistemas vivos. Describe los patrones de comportamiento en el desarrollo cíclico de las empresas, así como la capacidad de estas últimas para diagnosticar sus propias decisiones estratégicas, incluidas las instrucciones sobre cómo impulsar una empresa a un éxito a largo plazo.

Fuente: elaborado a partir de Montoya et al. (2020)

\section{MARKETING SIMBIÓTICO}

Los aportes de los autores llevan a establecer el concepto del marketing simbiótico como un proceso de marketing que se lleva a cabo en coordinación con otras organizaciones de tal forma que se puedan tener ventajas sinérgicas del proceso. Entre los beneficios destacados se encuentran la posibilidad de creación de marca (co-branding), de procesos de producción (co-producción), de relaciones de producción circulares, aprovechamiento de desarrollo conjunto con los clientes, desarrollo de nuevos nichos de mercado, explotación de competencias individuales organizacionales que se ponen a disposición de las otras organizaciones, apoyos 
financieros, colaboración para innovación y desarrollo de nuevos productos y servicios que se encuentra en casi todas las industrias, pero en especial, las de transporte y turismo, en las que la cooperación resulta indispensable para alcanzar los objetivos organizacionales.

Para Townsend (2003) es importante entender el paradigma de la relación, en especial, al tomar como base las motivaciones y relacionar la estructura de las alianzas con la cultura organizacional individual de cada una de las empresas, de tal forma que se obtengan de acuerdo con las características de la gestión y el desempeño. El gráfico 8 señala que el principal paradigma de la relación de la integración organizacional se fundamenta en los motivos, estructura, gestión, desempeño y cultura organizacional que se integran para lograr la salida deseada de ventajas competitivas sustentables.

\section{Gráfico 8. Conceptualización de la alianza}
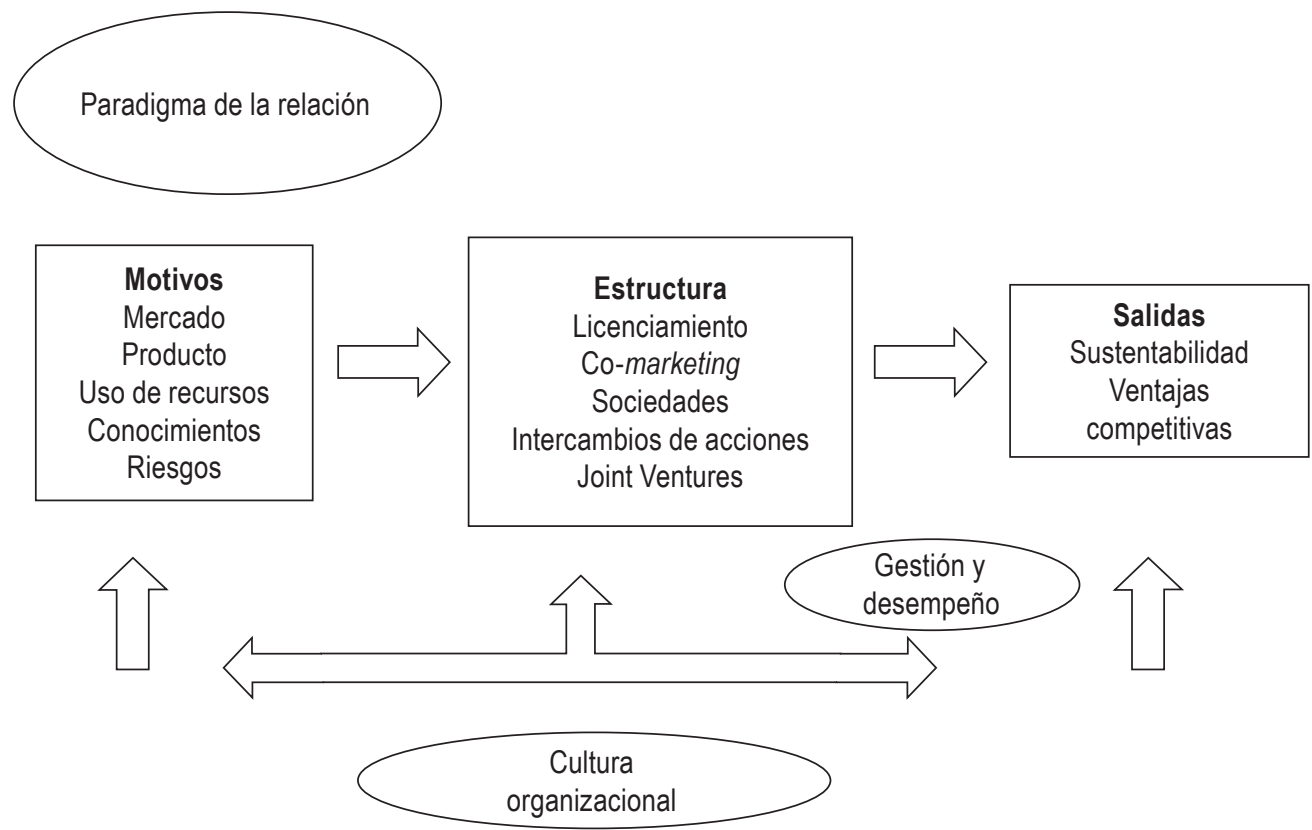

Fuente: Townsend (2003, p. 152)

De igual forma, las condiciones para la relación de tipo simbiótico en los procesos de marketing se pueden enmarcar en unas características que facilitan el proceso y que deben tener claridad al momento de iniciar el proceso de cooperación, no importa cuántas áreas organizacionales se encuentren involucradas (tabla 2). 
Tabla 2. Condiciones del marketing simbiótico

Característica

Característica

1. Grado de interacción

2. Motivación de la relación

Es el grado de compromiso de las organizaciones en la relación simbiótica, el nivel de confianza, la dificultad de las tareas de integración y los niveles organizacionales comprometidos, permiten analizar el grado de interacción. A mayor grado de interacción se encuentran mayores sinergias.

Las interacciones consumen recursos y proporcionan capacidades a las organizaciones, de allí la importancia que sean, no solo eficientes sino efectivas también.

La claridad de los objetivos por los cuales las organizaciones realizan la cooperación es fundamental. Las motivaciones deben ser coherentes, realizables y tener claridad sobre las expectativas. Entre más motivaciones tengan las áreas relacionadas, mayor posibilidad de éxito.

La duración de la simbiosis deberá estar bien definida, puede ser en un tiempo determinado o por proyecto u objetivo.

3. Temporalidad y permanencia de También se debe indicar si es una relación a corto plazo o la relación por una sola ocasión, o si se requiere un plazo medio, largo o incluso indefinido. La relación es más confiable y exitosa entre más duradera.

Medida de cuántas unidades de negocio o departamentos

4. Cantidad de unidades comprome- se encuentran involucrados, así como unidades geográficas tidas en la relación o estratégicas. De igual forma, si son unidades funcionales o basadas en actividades específicas.

La relación es inter - o intra-funcional, determina qué unida-

5. Especialidad en la relación des de la organización se requieren y si se necesitan apoyos complementarios.

Define si se requiere volver la relación institucional, si las re-

6. Normatividad de la relación laciones son de carácter tácito o explícito Se encuentra regulada por normas escritas o contratos.

Existe algún tipo de liderazgo por parte de algunas de las or-

7. Independencia de los agentes ganizaciones, las organizaciones son complementarias o competitivas.

Si las organizaciones son competencia, complementarias o per-

8. Similaridad de las organizaciones tenecen a otros sectores; el tamaño, experiencia, recursos y capacidades de las organizaciones; si son similares o diferentes.

Se describe si las organizaciones pertenecen al mismo siste-

9. Estructura de la cadena de valor ma de cadena de valor, su participación en el proceso y de ahí, sus requerimientos y necesidades.

Qué requisitos de tipo tecnológico se requieren para garanti-

10. Apoyos tecnológicos zar la simbiosis dependen también de la ubicación geográfica y de las motivaciones de la estrategia cooperativa. 
Marketing simbiótico. Una aproximación al concepto desde una revisión teórica

Característica

Definición

El nivel de cooperación y las necesidades son co-diseño, co-

11. Tipos de cooperación: diseño, producción, crowdfounding y dependiendo del modelo elegiproducción, mercadeo. do, las características serán variadas. Se deben establecer claramente las ventajas de la simbiosis en cada nivel de la cadena de valor.

12. Compromiso de la relación

Qué tan importante resulta para las organizaciones la simbiosis, establecer si es posible que esta genere actividades adaptativas y que sean recíprocas.

Modificar el entorno a partir del modelo simbiótico es una alternativa poderosa en este tipo de organización, se debe establecer claramente cuáles son los recursos y capacidades

13. Capacidad de crear ventajas con- compartidos y cuáles de ellos emergen como sinergias juntas deseables. Lo ideal es que todas las organizaciones adquieran las competencias que se desarrollan de la relación, de tal forma que el mutualismo se entienda como un mejoramiento para cada una de las partes.

El nivel jerárquico que toma las decisiones será fundamental

14. Integración en la toma de decisio- en este proceso, lo ideal es que el compromiso sea siempre nes y flexibilidad organizacional desde la alta gerencia, pero debe ser a su vez, compartido por todos los miembros del equipo.

Algunas organizaciones, por su estructura y organización, requieren niveles de formalización de las alianzas, nuevas alternativas organizacionales que van surgiendo a la par de la institucionalidad, entre ellas cadenas productivas, ecosistemas organizacionales, alianzas productivas o estratégicas, entre otras.

Depende del grado de similitud y de los niveles de recur-

16. Complementariedad o no comple- sos que tengan las organizaciones, se encontrarán alternatimentariedad de recursos vas de complementariedad o no, dependiendo de los objetivos de la simbiosis.

17. Congruencia de objetivos y valoLos resultados de las simbiosis deben ser congruentes con los objetivos organizacionales de cada una de las organizaciones involucradas, aunque sean de nivel o impacto diferente.

Fuente: Montoya et al. (2020)

\section{CONCLUSIÓN}

Las empresas -en especial las empresas pequeñas- buscan procesos colaborativos para mejorar su desempeño en entornos cambiantes y complejos. Sin embargo, las relaciones colaborativas no son fáciles de entender y gestionar, requieren de mucha confianza y compromiso entre las partes (Montoya et al., 2020), y aunque se debe tener en cuenta que casi todas las relaciones empresariales son simbióticas ya que las dos partes obtienen un beneficio (Varadarajan y Rajaratnam, 1986), 
realizar esfuerzos de manera consciente para cooperar y lograr que todas las partes involucradas obtengan un beneficio debe ser un ejercicio planeado y estructurado que requiere esfuerzo, cambio de entornos culturales y modelos de cooperación, incluso a veces complejos.

El marketing simbiótico se evidencia en una red de cooperación entre empresas que tienen como objetivo trabajar juntos y permitir el establecimiento de relaciones sociales que contribuyen a la proximidad y establecimiento de confianza entre ellos (Donato et al., 2017). Estos procesos de co-creación de valor como estrategia administrativa posibilita el fortalecimiento de las marcas y la consolidación de mercados, la cooperación incluso trasciende las áreas funcionales de mercadeo o las unidades estratégicas de negocio, buscan incluso otros tipos de cooperación financiera o de desarrollo y producción. Retomar el concepto de simbiosis permite -mediante la metáfora biológica de Montoya et al., (2012)- favorecer el entendimiento de los procesos cooperativos, que hoy en día, en entornos complejos virtuales, tiene más oportunidades y favorece procesos en tiempos y lugares diferentes; el futuro se visibiliza como simbiosis en línea, con posibilidades inmensas para mejorar los procesos en las organizaciones.

Este recorrido por los diferentes autores sobre los conceptos de marketing simbiótico permite analizar que si bien es un tema que no se ha desarrollado con la fuerza que tienen otros temas de mercadeo, ha permanecido constante desde su surgimiento en los años sesenta. La aplicación del concepto se ha dado en diferentes escenarios, en los cuales los procesos "co" (de cooperación, colaboración y trabajo conjunto) resultan oportunos al demostrar mayor eficiencia que el trabajo organizacional aislado. Se destaca que los autores resaltan las bondades de los procesos integrativos, se señalan por supuesto, algunos retos importantes como la necesidad de garantizar ganancias a cada uno de los integrantes del proceso de inter-relación, en especial, que mantengan su independencia e integridad y logren realmente la simbiosis como modelo propuesto.

\section{REFERENCIAS}

Abou-Shouk, Mohamed.; Tamam, Mahmoud., y Hewedi, Mahmoud (2019). Adopting cooperative marketing strategy by tourism service providers: comparing perceptions of travel agencies and hotels. En: Ekinci, Yucksel; Sharples, Liz; Viglia, Giampalo y Dogan Gursoy. 9th $^{\text {th }}$ advances in hospitality and tourism marketing and management conference. Portsmouth, United Kingdom: University of Portsmouth, p.119-131.

Acquire, Aurélien ;Carbone, Valentina, y Massé David (2019). How to create value (s) in the sharing economy: Business models, scalability, and sustainability. En: Technology Innovation Management Review, Vol. 9 N. ${ }^{\circ}$ 2, p. 5-24 Doi: 10.22215/timreview/1215 
Adler, Lee. (1966). Symbiotic marketing. En: Harvard Business Review, Vol. 44, N. ${ }^{\circ}$ 6, p. 59-71.

Ahn, SooKyoung; Kim, Hae; Forney Judith (2010). A Fashion collaboration or collision? Examining the match-up effect in co-marketing alliances. En: Journal of Fashion Marketing and Management: An International Journal, Vol. 14, N. ${ }^{\circ}$ 1, p. 6-20. DOI: https://doi. org/10.1108/13612021011025401

Brun, Juliette (2018). Symbiotic innovation: towards organizational structures relying on knowledge interdependency to foster the innovation process. Earlybird - Baltimore, United States: EURAM 2018 (European Academy of Management) Conference, p. 1-24.

Bucklin, Louis, y Sengupta, Sanjit (1993). Organizing successful co-marketing alliances. En: Journal of Marketing, Vol. 57, N. ${ }^{\circ}$ 2, p. 32-46. DOI: https://doi.org/10.2307/1252025

Cap, Jan-Patrick Blaich, Erik., Kohl, Holger, Von Raesfeld, Ariane., Harms, Rainer, y Will, Markus (2019). Multi-level network management-A method for managing inter-organizational innovation networks. En: Journal of Engineering and Technology Management, Vol. 51, p. 21-32. DOI: https://doi.org/10.1016/j.jengtecman.2019.02.001

Castellanos Oscar, Fuquene, Mayerli, Ramírez Diana. 2011. Análisis de tendencias de la información hacia la innovación. Bogotá: Programa Interdisciplinario de Investigación y Desarrollo en Gestión, Productividad y Competitividad. Bogotá, Colombia: Universidad Nacional de Colombia. 208 p.

Cegarra, Jean-Jack y Michel, Géraldine (2003). Alliances de marques: quel profit pour les marques partenaires? En: Revue Française de Gestion, N. ${ }^{\circ}$ 145, p.163-174.

Cherubini Sergio, Canigiani Marco. (1999). Il co-marketing sportivo. Strategie di cooperazione nel mercato sportivo. Italia: Franco Angeli. 112 p.

De Vita, Paolo. (2000). Organizzare il co-marketing. En: Sviluppo y Organizzazione, N. ${ }^{\circ}$ 181, p.37-54.

Dickinson, Joshua (2012). Symbiotic marketing: A network perspective. En: Journal of Management and Marketing Research, N. ${ }^{\circ} 11$, p.1-27.

Donato, Hellen Claudia; Farina, Milton Carlos; Donaire, Denis y Santos, Isabel Cristina Dos (2017). Value Co-Creation And Social Network Analysis On A Network Engagement Platform. En: Ram, Rev. Adm. Mackenzie, Vol. 18, N. ${ }^{\circ}$ 5, p. 63-91. DOI: http://dx.doi.org/10.1590/16 $\overline{78}$ 69712017/administracao.v18n5p63-91

Doyle, Peter (1995). Marketing in the new millennium. En: European Journal of Marketing, Vol. 29, N. ${ }^{\circ}$ 13, p. 23-41. DOI: https://doi.org/10.1108/03090569510147712

Farrelly, Francis., y Quester, Pascale. (2005). Investigating large-scale sponsorship relationships as co-marketing alliances. En: Business Horizons, Vol. 48, №. 1, p. 55-62.

Grieco, Cecilia y Lasevoli, Gennaro. (2017). Co-marketing alliances: definitions and approaches. Insights from a literature review. En: Management Research Review, Vol. 40, №. 9, p. 971-989. DOI: 10.1108/MRR-11-2016-0270

Hansen, Birgitte Gorm (2011). Beyond the boundary: Science, industry, and managing symbiosis. En: Bulletin of Science, Technology y Society, Vol. 31, N. ${ }^{\circ}$ 6, p. 493-505. DOI: https://doi. $\overline{\text { org }} / 10.1177 / 0270467611422834$ 
Huang, Jun, Li, Ji., Zhang, P., Cai, Zhenyao y Wang, Xinran (2011). Symbiotic marketing and trust-related issues: Empirical evidence from an emerging economy. En: Journal of Global Marketing, Vol. 24, N. ${ }^{\circ}$, p. 417-432. DOI: https://doi.org/10.1080/08911762.2011.634326

Iasevoli, Gennaro (2004) La valutazione della coerenza tra" brand partners" nelle alleanze di marketing. En: Micro \& Macro Marketing, Vol. 13, N. ${ }^{\circ}$ 3, p. 645-662. DOI: 10.1431/18867

Ketonen-Oksi, Sanna; Valkokari, Katri. Innovation (2019) Ecosystems as Structures for Value Co-Creation. En: Technology Innovation Management Review, Vol. 9, N. 2, p. 25-35.

Kiran Vasanth, Majumdar, Mousumi Kishore Krishna (2013). Symbiotic marketing - the conceptual dimensions. En: Asia Pacific Journal of Marketing and Retail Management, Vol. 4, p.1-10.

Li, J., Hu, Yanghong., Zhang, Lan y Sun, Wei. (2018). Symbiosis-Orientation and the Survival of Small-Large Strategic Alliances. En: Journal of Entrepreneurship and Innovation in Emerging Economies, Vol. 4, N. 2, p. s121 $\overline{136}$. DOI: https://doi.org/10.1177/2393957518778475

Lockett, Andy, y Blackman, Ian. (2001). Strategies for building a customer base on the Internet: symbiotic marketing. En: Journal of Strategic Marketing, Vol. 9, N. ${ }^{\circ}$ 1, p. 47-68. DOI: https://doi. org/10.1080/7137757 $\overline{27}$

Lorek, Elzbieta y Lorek, Agnieszka (2020). A Sustainable Business Model in the Functioning of Enterprises as the Base for Creating Circular Economy: The Present and Development Prospects. En: In Networked Business Models in the Circular Economy. Pensilvania, Estados Unidos: IGI Global, p. 54-81.

Mikulášková Justína, Čambál Miloš, Polakovič L’uboš, Urbanovičová Petra. (2020). Spiral Management: New Concept of the Social Systems Management. En: Networked Business Models in the Circular Economy. Pensilvania, Estados Unidos: IGI Global, p. 174-199.

Montoya, Alexandra y Montoya, Iván (2012). Metáforas biológicas aplicadas a las organizaciones. Universidad Nacional de Colombia. Bogotá, Colombia: Facultad de Ciencias Económicas. Centro de Investigaciones para el Desarrollo (CID), 50 p.

Montoya, Alexandra, Montoya, Iván y Valencia, Alejandro (2016). Metáforas biológicas aplicadas a las organizaciones II: ensayos escogidos. Bogotá, Colombia: Universidad Nacional de Colombia, Facultad de Ciencias Económicas, 122 p.

Montoya, Alexandra, Montoya, Iván y Castellanos Oscar. (2010). La metáfora organizacional: alternativa de entendimiento procedente de otras ciencias. En: Revista Facultad de Ciencias Económicas: Investigación y Reflexión, Vol. 18, N. ${ }^{\circ}$ 1, p. $75 \overline{-86}$.

Montoya, Alexandra. Montoya, Iván. (2012). Aplicación de la metáfora biológica para el desarrollo de formas organizativas en la integración empresarial. En: Revista Facultad de Ciencias Económicas: Investigación y Reflexión, Vol. 20, N. ${ }^{\circ}$ 2, p. 43-54. DOI: https://doi.org/10.18359/ rfce. 2163

Montoya Alexandra. Montoya Iván. Castellanos Oscar. (2020) Aplicaciones metafóricas en gestión de organizaciones. Bogotá, Colombia: Universidad Nacional de Colombia. Working paper.

Moraitis, Emmanuil. (2019). Symbiotic synergies: adaptive framework for polydisciplinary collaboration in performance practice (Doctoral dissertation. Salford, UK: University of Salford, 166p. 
Moriarty, David E. y Mikkulainen, Risto (1996). Efficient reinforcement learning through symbiotic evolution. En: Machine learning, Vol. 22, №.1-3, p.11-32.

Muñoz, Pablo., y Cohen, Boyd. (2018). A Compass for Navigating Sharing Economy Business Models. En: California Management Review, Vol. 61, N. ${ }^{\circ}$ 1, p.114-147. DOI: https://doi. org/10.1177/0008125618795490

Pinarbasi, Faith. (2020). Sharing Economy and Applications: Business and Marketing Perspective. In Networked Business Models in the Circular Economy. Pensilvania, Estados Unidos: IGI GLOBAL, p. 82-102.

Prahalad, Coimbatore y Ramaswamy, Venkat (2004). Co-creation experiences: The next practice in value creation. En: Journal of Interactive Marketing, Vol. 18, N. ${ }^{\circ}$ 3, p. 5-14.

Robinson, Richard; Baum, Tom; Golubvskaya, Maria; Solne, David; y Callan Victor (2019). Applying endosymbiosis theory: Tourism and its young workers. En: Annals of Tourism Research, Vol. 78, No. 102751, p. 1-12. https://doi.org/10.1016/j.annals.2019.102751

Shaw, Colin (2013). Collaboration is dead - long live symbiosis. Beyond Philosophy.

Skarzauskiene, Aelita; Maciuliene, Monika; Ivanova-Radovanova, Petja (2019). Modelling Co-creation Ecosystem for Public Open Spaces. En: CyberParks-The Interface Between People, Places and Technology. Berlín, Alemania: Springer, p. 262-277.

Shimizu, Koichi (2003). Symbiotic Marketing Strategy. Tokyo, Japan: Souseisha Book Company.

Taek Yi, Ho; Lee, Joseph; y Dubinsky, Alan. (2010). An empirical investigation of relational conflicts in co-marketing alliances. En: Journal of Business-to-Business Marketing, Vol. 17, N. ${ }^{\circ}$ 3, p. 249-278. DOI: https://doi.org/10.1080/10517120903465350

Thomas, Robert J., y Yoram Jerry Wind. (2013). Symbiotic innovation: getting the most out of collaboration. En: Evolution of Innovation Management, p. 1-31.

Thoumrungroje, Amonrat, y Patriya Tansuhaj. (2004). Globalization effects, co-marketing alliances, and performance. En: Journal of American Academy of Business, Vol. 5 N. ${ }^{\circ}$ 1/2, p. 495-502.

Townsend, Janell (2003). Understanding alliances: a review of international aspects in strategic marketing. En: Marketing Intelligence y Planning, Vol. 21, N. ${ }^{\circ}$, p. 143 155. DOI: http://dx.doi. org/10.110 $\overline{8 / 0} 2634500310474966$

Udaya, Kumar (1994). https://shodhganga.inflibnet.ac.in/handle/10603/905. India: Pondicherry University, 369p.

Varadarajan, Rajan; Cunningham, Margaret H. (1995). Strategic alliances: a synthesis of conceptual foundations. En: Journal of the Academy of Marketing Science, Vol. 23, N. 4, p. 282. DOI: https://doi.org/10.1177/009207039502300408

Varadarajan, Rajan, Jayachandran. Satish (1999). Marketing strategy: an assessment of the state of the field and outlook. En: Journal of the Academy of Marketing Science, Vol. 27, N. 2, p. 120-143. DOI: https://doi.org/10.1177/0092070399272002

Varadarajan, Rajan y Rajaratnam, Daniel. (1986). Symbiotic marketing revisited. En: Journal of Marketing, Vol. 50, N. ${ }^{\circ}$ 1, p.7-17. DOI: https://doi.org/10.1177/002224298605000101 
Varelas, Sotiris., Kavoura, Androniki, Giovanis, Apostolos., Borges-Tiago, Teresa., y Tiago, Flavio. (2019). Strategic Synergies and Co-marketing: A Case Study of the Airline Sector. En: Kavoura Androniki., Kefallonitis, Efstathios., Giovanis Apostolos..Strategic Innovative Marketing and Tourism. Berlín, Alemania: Springer, p. 629-635.

Venkataraman, Venkat (1981). Marketing Synergy: A Key to Increased Productivity. En: Productivity, Vol. 22, N. ${ }^{\circ}$ 1, p. 7-12.

Venkatesh, Raghavan; Mahajan, Vijay; y Muller, Eitan (2000). Dynamic co-marketing alliances: When and why do they succeed or fail? En: International Journal of Research in Marketing, Vol. 17, N. ${ }^{\circ}$ 1, p.3-31. DOI: https://doi.org/10.1016/S0167-8116(00)00004-5

Vyacheslav Yukalov, Yukalova, y Sornette, Didier (2012). Modeling Symbiosis by Interactions Through Species Carrying Capacities. En: Physica D: Nonlinear Phenomena, Vol. 241, p. 1270-1289. DOI: https://doi.org/10.1016/j.physd.2012.04.005

Zaheer, Akbar., y Venkatraman, Venkat. (1995). Relational governance as an interorganizational strategy: An empirical test of the role of trust in economic exchange. En: Strategic Management Journal, Vol. 16, N. ${ }^{\circ}$, p.373-392. DOI: https://doi.org/10.1002/smj.4250160504 